\title{
Editorial
}

\section{New online submission system and acknowledgements}

\section{Dear Readers, Contributors and Reviewers,}

As previously announced, ScholarOne will be used as of January 7,2013 , as our online submission system. Our whole team is enthusiastic with the new system. We advise all the authors to carefully read the instructions for authors before starting the submissions.

It is a good time to thank all reviewers and authors. We also have to thank the National Council for Scientific and Technological Development (CNPq) and the University of São Paulo for the financial support. We always emphasize the Journal of Applied Oral Science is published by our dental school, a non-profit making institution, so that the support from the federal and state governments is the only financial source to our journal.
Please feel free to contact us through our e-mail address: jaos@usp.br. We also invite all readers, contributors and reviewers to visit our free complete online collection at http://www.scielo.br/jaos.

Finally, we take advantage of this opportunity to wish all of you Happy Holidays!

With our best regards,

\section{Carlos F. Santos}

DDS, MSc, PhD, Professor

Editor-in-Chief

Journal of Applied Oral Science 